\title{
Comparison of neoadjuvant chemoradiation with carboplatin/ paclitaxel or cisplatin/5-fluoruracil in patients with squamous cell carcinoma of the esophagus
}

Stefan Münch ${ }^{1,2^{*}}$, Steffi U. Pigorsch ${ }^{1,2}$, Marcus Feith ${ }^{3}$, Julia Slotta-Huspenina ${ }^{4}$, Wilko Weichert ${ }^{2,4}$, Helmut Friess ${ }^{3}$, Stephanie E. Combs $s^{1,2,5}$ and Daniel Habermehl $1^{1,2,5^{*}}$

\begin{abstract}
Purpose: Neoadjuvant chemoradiation (nCRT) is the treatment of choice for patients with locally advanced squamous cell carcinoma of the esophagus (SCC). Today radiation oncologists can choose between two different therapy regimes including chemoradiation with cisplatin and 5-fluoruracil (CDDP/5FU) and chemoradiation analogue to the CROSS-regime with carboplatin and paclitaxel (Carb/TAX). However, there is a lack of studies comparing these regimes, especially for the subgroup of patients with SCC. In this study, we want to compare nCRT with CDDP/5FU and nCRT with Carb/TAX for patients with locally advanced SCC.

Patients and methods: We retrospectively compared 20 patients who were scheduled for nCRT with a total radiation dose of 41.4 Gy (daily dose of 1.8 Gy) and weekly chemotherapy with carboplatin (Area under the curve 2) and Paclitaxel (50 mg per square meter of body-surface area) according to the CROSS-regime to 31 patients who were scheduled for nCRT with a total radiation dose of 45 Gy (daily dose of $1.8 \mathrm{~Gy}$ ) and simultaneous chemotherapy with cisplatin (20 mg/ $\mathrm{m}^{2} / \mathrm{d}$ ) and 5-fluoruracil (500 mg/m²/d) on day 1-5 and day 29-33. For the per-protocol (PP) analysis, per protocol treatment was defined as either complete radiation with $41.4 \mathrm{~Gy}$, at least three complete cycles of Carb/TAX and subsequent surgery or complete radiation with $45 \mathrm{~Gy}$, at least one complete cycle of CDDP/5FU and subsequent surgery.

Results: Fifty-one patients (31 patients treated with CDDP/5FU and 20 patients treated with Carb/TAX) were evaluated for the intention-to-treat (ITT) analysis and 44 patients (26 patients treated with CDDP/5FU and 18 patients treated with Carb/TAX) were evaluated for the PP analysis. No significant differences were seen for baseline and tumor characteristics like age, sex, TNM-stage, grading and tumor extension between patients treated with Carb/TAX and patients treated with CDDP/5FU. The most common tumor regression grade after nCRT was grade I as classified by Becker et al., which was observed in 84 and $79 \%$ of patients. No significant differences in tumor regression grades were seen between both regimes. Postoperative insufficiency of the anastomosis was seen in 6 patients (33\%) who were treated with Carb/TAX and 4 patients (15\%) who were treated with CDDP/5FU $(p=0.273)$. Patients treated with CDDP/ 5FU developed significantly more cumulative hematologic III' (CTCAE) toxicities (58\% vs 20\%; $p=0.010$ ) than patients treated with Carb/TAX. In contrast to that, there was no significant difference for overall survival (OS) and freedom from relapse (FFR) between treatment groups.

(Continued on next page)
\end{abstract}

\footnotetext{
*Correspondence: stefan.muench@mri.tum.de; daniel.habermehl@tum.de

${ }^{1}$ Department of Radiation Oncology, Klinikum rechts der Isar, Technical

University Munich, Ismaninger Str. 22, D-81675 Munich, Germany

Full list of author information is available at the end of the article
}

(c) The Author(s). 2017 Open Access This article is distributed under the terms of the Creative Commons Attribution 4.0 International License (http://creativecommons.org/licenses/by/4.0/), which permits unrestricted use, distribution, and reproduction in any medium, provided you give appropriate credit to the original author(s) and the source, provide a link to the Creative Commons license, and indicate if changes were made. The Creative Commons Public Domain Dedication waiver (http://creativecommons.org/publicdomain/zero/1.0/) applies to the data made available in this article, unless otherwise stated. 
(Continued from previous page)

Conclusion: In this retrospective analysis, no significant difference was seen for OS and FFR between nCRT with CDDP/ 5FU and nCRT with Carb/TAX. However, the application of CDDP/5FU was associated with significantly more hematologic III - toxicities compared to Carb/TAX. Future prospective trials should investigate if these results are reproducible in randomized patient cohorts.

Keywords: Squamous cell carcinoma of the esophagus, Neoadjuvant chemoradiation, Cisplatin/5-fluoruracil, Carboplatin/Paclitaxel

\section{Introduction}

With 400.000 estimated deaths and 456.000 new cases in 2012 esophageal cancer (EC) is the sixth most common cause of cancer death and the eighth most common cancer in the world [1].

In 2008, Tepper et al. [2] demonstrated the superiority of neoadjuvant chemoradiation (nCRT) with cisplatin and 5-fluoruracil (CDDP/5FU) combined with subsequent surgery compared to surgery alone. Multimodal treatment was associated with a significant increase in terms of overall survival and progression-free survival and therefore nCRT with CDDP/5FU and subsequent resection became the treatment of choice for patients with locally advanced squamous cell carcinoma of the esophagus (SCC) suitable for surgery.

However, in the more recent CROSS-study, van Hagen et al. [3] compared nCRT with carboplatin and paclitaxel (Carb/TAX) and subsequent surgery to surgery alone in patients with locally advanced EC. Comparable to the results of Tepper et al. patients treated with nCRT with Carb/TAX had a significantly improved overall survival and disease-free survival compared to patients treated with surgery alone. In addition, van Hagen et al. performed a subgroup analysis for patient with SCC and adenocarcinoma (AC) of the esophagus. Thereby, the effect of nCRT was much higher for patients with SCC than for patients with AC.

At out department nCRT with CDDP/5FU was the treatment of choice for locally advanced SCC until 2014. Since then patients with locally advanced SCC were treated analogous to the CROSS-trial. Until now there is only one retrospective analysis comparing nCRT with CDDP/5FU and nCRT with Carb/TAX for patients with EC [4]. In the mentioned study, patients treated with $\mathrm{CDDP} / 5 \mathrm{FU}$ had more hematological toxicities, whereas there was no significant difference for overall survival. However, approximately $75 \%$ of patients included in this study had AC, while only $24 \%$ of patients had SCC. Because the effect of nCRT on overall survival is higher in patients with SCC compared to patients with $\mathrm{AC}$, there might be a difference in efficiency between nCRT with CDDP/5FU and nCRT with Carb/TAX in SCC patients.
In order to clarify if there should be a preferred chemotherapy regime for SCC patients this study evaluates and compares toxicity and oncological outcome parameters of these two different nCRT regimes in a homogenous group of SCC patients.

\section{Patients and methods}

Between 2011 and 2016, 51 patients with locally advanced SCC were initially recruited for nCRT with either CDDP/5FU or Carb/TAX at our Department.

\section{Treatment groups}

We retrospectively compared 20 patients who qualified for nCRT with radiotherapy up to a total dose of 41.4 Gy (daily doses of $1.8 \mathrm{~Gy}$ ) and concomitant, weekly chemotherapy with carboplatin (area under the curve 2) and Paclitaxel (50 mg per square meter of body-surface area) according to the CROSS-regime [3] to 31 patients who were recruited for nCRT with a total radiation dose of 45 Gy (daily dose of 1.8 Gy) and a simultaneous chemotherapy with cisplatin $\left(20 \mathrm{mg} / \mathrm{m}^{2} / \mathrm{d}\right)$ and 5fluoruracil $\left(500 \mathrm{mg} / \mathrm{m}^{2} / \mathrm{d}\right)$ on day $1-5$ and $29-33$.

For the per-protocol analysis (PP), per protocol treatment was defined as either complete radiation with 41.4 Gy and at least three cycles of Carb/TAX and subsequent surgery or complete radiation with $45 \mathrm{~Gy}$ and at least one cycle of $\mathrm{CDDP} / 5 \mathrm{FU}$ with subsequent surgery. In summary 7 of 51 patients (14\%) were excluded for the per-protocol analysis. One patient of the Carb/TAX group chemotherapy was switched to CDDP/5FU after the first cycle due to an allergic reaction and another one was lost to follow-up before surgery. Within the CDDP/5FU arm one patient was excluded because he received chemotherapy with only cisplatin due to myelodysplastic syndrome and another patient was excluded because the RT protocol was switched to 41.4 Gy due to a high mean lung dose. In one patient therapy was terminated when reaching 27 Gy due to an esophagotracheal fistula and one additional patient did not undergo surgery due to systemic progression. Another patient was excluded because he did not receive at least $50 \%$ of chemotherapy. 


\section{Radiotherapy}

All patients in the Carb/TAX group were treated with volumetric modulated arc therapy (VMAT) while 22 patients (71\%) in the CDDP/5FU arm were treated with VMAT and 9 patients (29\%) were treated with 3-dimensional conformal radiotherapy (3D-CRT). VMAT was done with a median of two arcs [range 1-3] and 3D-CRT was done with a median of 5 [range 5-7] beams.

\section{Pathology}

Resected specimen were available for all 48 patients (94\%) who underwent surgery. If no tumor cells reached the margin, the resection was considered as complete and - since in all cases no other metastatic tumor manifestations were present - classified as R0. Response to nCRT was evaluated by extensive and standardized histomorphological workup of resection specimen as described by Becker et al. [5]. In this classification complete tumor regression with $0 \%$ residual tumor is classified as grade 1a, subtotal tumor regression with $<10 \%$ residual tumor per tumor bed is classified as grade $1 \mathrm{~b}$, partial tumor regression with $10-50 \%$ residual tumor per tumor bed is classified as grade 2 and minimal or no tumor regression with $>50 \%$ residual tumor per tumor bed is classified as grade 3. WHO grading of tumors (G1, G2, G3) was done on pretherapeutic biopsies.

\section{Follow- up}

Patients were regularly invited to follow-up examinations starting approximately 6 weeks after end of nCRT.

\section{Toxicity}

Acute side effects were retrospectively reviewed using medical records and classified according to the Common Terminology Criteria for Adverse Events (CTCAE) v. 4.03.

\section{Statistics}

In the first step, we performed an intention-to-treat analysis (ITT) with all patients who were scheduled for one of the mentioned therapy regimes. To consider protocol violations we then performed a PP analysis.

Freedom from relapse (FFR) was calculated for patients who underwent surgery. The respective time interval was defined from the day of surgery until tumor progression or tumor recurrence. Overall survival (OS) was defined as the time interval from the beginning of treatment until death. Statistical analyses comprised comparison of baseline parameters, side effects, and different dose parameters using the Wilcoxon-Mann-Whitney U test or Fishers exact test. OS and FFR where compared using the log-rank test. All statistical tests were conducted in an exploratory manner on two-sided 5\% significance levels using the software SPSS Statistics 18 version 18.0.0 (IBM SPSS Statistics, Armonk, U. S.).

\section{Results}

\section{Baseline and tumor characteristics}

Baseline and tumor characteristics for patients included in the ITT and PP analysis are presented in Table 1. Median age of patients was 62 years in both treatment groups and almost two third of patients were male. A total of $75 \%$ of all patients in the Carb/TAX group and $87 \%$ of all patients in the CDDP/5FU group had uT3 tumors and all except one patient had suspected lymph node metastases in the initial endosonographic staging. Distant metastasis (lung) was seen in one patient of each group. In both of these patients the lung metastases were resected before initiation of nCRT. Median tumor extension was $5 \mathrm{~cm}$ (IQR 3-6). All except one patient had moderately (G2, 54\%) or poorly differentiated (G3, $44 \%)$ tumor grading. Tumor extension was not comprehensible in 2 patients, while grading was not comprehensible in three patients. Median time between end of nCRT and surgery was 38 days [range 9-86 days]. In summary, no significant differences in baseline parameters were seen between patients treated with Carb/TAX and patients treated with $\mathrm{CDDP} / 5 \mathrm{FU}$.

\section{Pathology}

Resection status was insecure (RX) in three patients (6\%), due to tissue artifacts induced by complex surgical procedures in complicated cases. However, while complete resection (R0) was achieved in 44 of the remaining 45 patients (98\%), in one patient (2\%) treated with Carb/TAX resection status was classified as R1. No significant difference was seen for the distribution of tumor regression grade after nCRT (Table 2). The most common tumor regression grade was grade I $(<10 \%$ residual tumor) which was seen in $84 \%$ of patients (ITT and PP) treated with the CROSS regime and 79\% (ITT) and $80 \%(\mathrm{PP})$ of patients treated with $\mathrm{CDDP} / 5 \mathrm{FU}$.

\section{Toxicities}

In one patient of the $\mathrm{CDDP} / 5 \mathrm{FU}$ group therapy was terminated when reaching 27 Gy due to an esophagotracheal fistula. No significant differences were seen for the proportion of patients suffering from postoperative insufficiency of the anastomosis. Referring to the perprotocol population 6 patients $(33 \%)$ who were treated with Carb/TAX and 4 patients (15\%) who were treated with CDDP/5FU suffered from insufficiency of the anastomosis $(p=0.273)$.

Regarding hematologic parameters significantly more grade III toxicities were seen in patients treated with 
Table 1 Patients' and tumor characteristics

\begin{tabular}{|c|c|c|c|c|c|c|}
\hline \multirow[t]{2}{*}{ Parameter } & \multicolumn{3}{|c|}{ Intention to treat } & \multicolumn{3}{|c|}{ Per protocol } \\
\hline & $\begin{array}{l}\text { Carb/TAX } \\
n=20\end{array}$ & $\begin{array}{l}\text { CDDP/5FU } \\
n=31\end{array}$ & $\overline{p \text {-value }}$ & $\begin{array}{l}\text { Carb/TAX } \\
n=18\end{array}$ & $\begin{array}{l}\text { CDDPs/5FU } \\
n=26\end{array}$ & $p$-value \\
\hline $\begin{array}{l}\text { Median Age } \\
\text { (IQR) }\end{array}$ & $62(56-71)$ & $62(55-72)$ & 0.875 & $61(56-69)$ & 65 (57-72) & 0.489 \\
\hline Male & $13(65 \%)$ & $20(65 \%)$ & 1.000 & $13(72 \%)$ & $16(62 \%)$ & 0.531 \\
\hline T-stage & & & 0.143 & & & 0.103 \\
\hline uT1 & $0(0 \%)$ & $1(3 \%)$ & & $0(0 \%)$ & $1(4 \%)$ & \\
\hline uT2 & $5(25 \%)$ & $2(6 \%)$ & & $5(28 \%)$ & $2(8 \%)$ & \\
\hline UT3 & 15 (75\%) & 27 (87\%) & & $13(72 \%)$ & $23(88 \%)$ & \\
\hline uT4 & $0(0 \%)$ & $1(3 \%)$ & & $0(0 \%)$ & $0(0 \%)$ & \\
\hline $\mathrm{uN}+$ & $20(100 \%)$ & $30(97 \%)$ & 1.000 & $18(100 \%)$ & 25 (96\%) & 1.000 \\
\hline CMO & 20 (100\%) & 31 (100\%) & 1.000 & $18(100 \%)$ & 26 (100\%) & 1.000 \\
\hline Grading & & & 1.000 & & & 0.765 \\
\hline G1 & $0(0 \%)$ & $1(3 \%)$ & & $0(0 \%)$ & $0(0 \%)$ & \\
\hline G2 & $11(58 \%)$ & 15 (52\%) & & $11(61 \%)$ & $14(56 \%)$ & \\
\hline G3 & $8(42 \%)$ & $13(45 \%)$ & & $7(39 \%)$ & $11(44 \%)$ & \\
\hline $\begin{array}{l}\text { Tumor extension } \\
\text { (IQR) }\end{array}$ & $4(2-6)$ & $5(3-7)$ & 0.233 & $4(2-6)$ & $5(3-6)$ & 0.232 \\
\hline
\end{tabular}

5-FU 5-fluoruracil, IQR inter-quartiles-range

$\mathrm{CDDP} / 5 \mathrm{FU}$ (58\%) compared to patients treated analogue to the CROSS-protocol $(20 \%)(p=0.010)$. Within the intention-to-treat population a leukopenia $\mathrm{I}^{\circ}, \mathrm{II}^{\circ}, \mathrm{III}^{\circ}$ and $\mathrm{IV}^{\circ}$ was observed in 20,50, 20 and $0 \%$ of patients treated with Carb/TAX, respectively. In contrast to that, leukopenia grade $\mathrm{I}^{\circ}, \mathrm{II}^{\circ}, \mathrm{III}^{\circ}$ and $\mathrm{IV}^{\circ}$ was seen in 13,19 , 48 and $10 \%$ of patients treated with CDDP/5FU $(p=0.065)$. In patients treated per protocol, significantly higher rates of leukopenia were seen in patients who received $\mathrm{CDDP} / 5 \mathrm{FU}$ than in patients who were treated with $\mathrm{Carb} / \mathrm{TAX}\left(\mathrm{I}^{\circ}: 15 \%\right.$ vs. $17 \%$, $\mathrm{II}^{\circ}$ : $19 \%$ vs. $56 \%$, $\mathrm{III}^{\circ}$ : $50 \%$ vs. $17 \%, \mathrm{IV}^{\circ}$ : $8 \%$ vs. $\left.0 \% ; p=0.048\right)$.

No significant differences were seen for thrombocytopenia $(p=0.654)$ and anemia $(p=0.364)$ between treatment groups. All hematologic side effects are shown in Table 3.

\section{In-hospital-mortality}

Two patients (12\%) in the Carb/TAX group and 3 patients (12\%) in the $\mathrm{CDDP} / 5 \mathrm{FU}$ group died before leaving the hospital after surgery $(p=1.000)$. Median time from surgery to death in these patients was 0.97 months. In three of five patients who died during hospitalization, death was caused by postsurgical acute respiratory distress syndrome without any signs for insufficiency of the anastomosis. One patient died from pulmonary complications due to an insufficiency of the anastomosis. The last patient died from sepsis with multiorgan failure, probably caused by an insufficiency of the anastomosis.

\section{Treatment failure}

In summary, local or distant treatment failure was seen in $4(22 \%)$ patients (Carb/TAX) and 8 (31\%) patients (CDDP/5FU) of the per-protocol population $(p=0.723)$ and in $4(21 \%)$ patients patients (Carb/TAX) and $10(34 \%)$ patients $(\mathrm{CDDP} / 5 \mathrm{FU})$ of the ITT-population $(p=0.354)$.

Table 2 Tumor regression grade

\begin{tabular}{|c|c|c|c|c|c|c|}
\hline \multirow{2}{*}{$\begin{array}{l}\text { Tumor } \\
\text { regression }\end{array}$} & \multicolumn{3}{|c|}{ Intention to treat } & \multicolumn{3}{|c|}{ Per protocol } \\
\hline & $\begin{array}{l}\text { Carb/TAX } \\
n=19\end{array}$ & $\begin{array}{l}C D D P / 5 F U \\
n=29\end{array}$ & $p$-value & $\begin{array}{l}\text { Carb/TAX } \\
\mathrm{n}=18\end{array}$ & $\begin{array}{l}C D D P / 5 F U \\
n=26\end{array}$ & $p$-value \\
\hline la (pCR) & $5(26 \%)$ & $11(38 \%)$ & 0.731 & $5(28 \%)$ & $10(38 \%)$ & 0.721 \\
\hline $\mathrm{lb}$ & 11 (58\%) & $12(41 \%)$ & & $10(56 \%)$ & $11(42 \%)$ & \\
\hline 2 & $1(5 \%)$ & $3(10 \%)$ & & $1(6 \%)$ & $3(12 \%)$ & \\
\hline 3 & $2(11 \%)$ & $3(10 \%)$ & & $2(11 \%)$ & $2(8 \%)$ & \\
\hline
\end{tabular}

$p C R$ pathologic complete remission 
Table $\mathbf{3}$ Hematologic toxicities

\begin{tabular}{|c|c|c|c|c|c|c|}
\hline \multirow{2}{*}{$\begin{array}{l}\text { Hematologic } \\
\text { toxicities }\end{array}$} & \multicolumn{3}{|c|}{ Intention to treat } & \multicolumn{3}{|c|}{ Per protocol } \\
\hline & $\begin{array}{l}\text { Carb/TAX } \\
n=20\end{array}$ & $\begin{array}{c}C D D P / 5 F U \\
n=31\end{array}$ & $p$-value & $\begin{array}{l}\text { Carb/TAX } \\
n=18\end{array}$ & $\begin{array}{l}C D D P / 5 F U \\
n=26\end{array}$ & $p$-value \\
\hline$\geq 1 \|^{\circ}$ & $4(20 \%)$ & $18(58 \%)$ & 0.010 & $3(17 \%)$ & $15(58 \%)$ & 0.012 \\
\hline Leukopenia & & & 0.065 & & & 0.048 \\
\hline $0^{\circ}$ & $2(10 \%)$ & $3(10 \%)$ & & $2(11 \%)$ & $2(8 \%)$ & \\
\hline $1^{\circ}$ & $4(20 \%)$ & $4(13 \%)$ & & $3(17 \%)$ & $4(15 \%)$ & \\
\hline$\|^{\circ}$ & $10(50 \%)$ & $6(19 \%)$ & & $10(56 \%)$ & $5(19 \%)$ & \\
\hline\|\|$^{\circ}$ & $4(20 \%)$ & $15(48 \%)$ & & $3(17 \%)$ & $13(50 \%)$ & \\
\hline $\mathrm{IV}^{\circ}$ & $0(0 \%)$ & $3(10 \%)$ & & $0(0 \%)$ & $2(8 \%)$ & \\
\hline Thrombocytopenia & & & 0.654 & & & 0.364 \\
\hline $0^{\circ}$ & 15 (75\%) & $18(58 \%)$ & & $14(78 \%)$ & $14(54 \%)$ & \\
\hline $1^{\circ}$ & $2(10 \%)$ & $6(19 \%)$ & & $2(11 \%)$ & $6(23 \%)$ & \\
\hline$\|^{\circ}$ & $2(10 \%)$ & $2(6 \%)$ & & $2(11 \%)$ & $2(8 \%)$ & \\
\hline$\| 1^{\circ}$ & $1(5 \%)$ & $4(13 \%)$ & & $0(0 \%)$ & $3(12 \%)$ & \\
\hline $\mathrm{IV}^{\circ}$ & $0(0 \%)$ & $1(3 \%)$ & & $0(0 \%)$ & $1(4 \%)$ & \\
\hline Anemia & & & 0.184 & & & 0.479 \\
\hline $0^{\circ}$ & $3(15 \%)$ & $0(0 \%)$ & & $2(11 \%)$ & $0(0 \%)$ & \\
\hline $1^{\circ}$ & $10(50 \%)$ & 19 (61\%) & & $10(56 \%)$ & $16(62 \%)$ & \\
\hline$\|^{\circ}$ & $6(30 \%)$ & $9(29 \%)$ & & $5(28 \%)$ & $7(27 \%)$ & \\
\hline\|\|$^{\circ}$ & $1(5 \%)$ & $3(10 \%)$ & & $1(6 \%)$ & $3(12 \%)$ & \\
\hline
\end{tabular}

Bold numbers indicate statistical significance with $p<0.05$

Loco-regional recurrence was the most common reason for treatment failure. Within the per-protocol population, loco-regional failure and distant failure was seen in 3 patients $(17 \%)$ and 2 patients $(11 \%)$ treated with Carb/TAXc, respectively. Compared to that, loco-regional failure and distant failure was seen in 6 patients $(23 \%)(p=0.716)$ and 3 patients $(12 \%)(p=1.000)$ treated with CDDP/5FU.

\section{Survival}

After a median follow-up of 34.6 months for surviving patients in the per-protocol population, median overall survival (OS) was 23.9 months for patients treated with Carb/TAX and 40.1 months for patients treated with CDDP/5FU. This difference was not statistically significant $(p=0.802)$ (Fig. 1$)$.

Median FFR was not reached in both patient groups. However, no significant difference was seen for patients treated with Carb/TAX or CDDP/5FU $(p=0.696)$ (Fig. 2).

\section{Discussion}

nCRT is the treatment of choice for patients with locally advanced SCC of the esophagus, suitable for surgery [6, 7]. Although different treatment protocols for nCRT have successfully improved overall survival and complete resection rates compared to surgery alone in EC patients, head-to-head comparisons with a competitive focus on oncologic efficacy and toxicity are still missing. The most widely used concomitant chemotherapy protocols are currently based on CDDP/ $5 F U$ or Carb/TAX. In this analysis, we retrospectively compared nCRT according to the CROSS-protocol to nCRT with CDDP/5FU in a slightly modified version of the regime described by Bates et al. [8].

While there were no differences regarding overall survival and freedom from relapse for the two treatment regimes, significantly more hematologic $\mathrm{III}^{\circ}$ toxicities were observed in patients treated with CDDP/5FU. These findings are consistent with the results of Blom et al. [4]. In their study, the authors compared nCRT analogue to the CROSS-regime with nCRT analogue to the regime published by Tepper et al. [2] for 165 patients with SCC or adenocarcinoma of the esophagus. No significant differences were seen for OS, but as in our study, patients treated with CDDP/5FU had a significantly higher risk of developing any grade III toxicity than patients treated with the CROSS-regime (41\% vs. $25 \%)$. Thereby, the most common $\geq \mathrm{III}^{\circ}$ toxicity was leukopenia, which was observed in $24 \%$ of patients treated with CDDP/5FU and $10 \%$ of patients treated with the CROSS-regime. In our study we also recognized a significant difference regarding the incidence of $\geq \mathrm{III}^{\circ}$ hematologic toxicities (58\% vs. $20 \%$, ITT and $58 \%$ vs. $17 \%$, PP). Especially for patients treated with Carb/TAX the rate of hematologic $\geq \mathrm{III}^{\circ}$ 


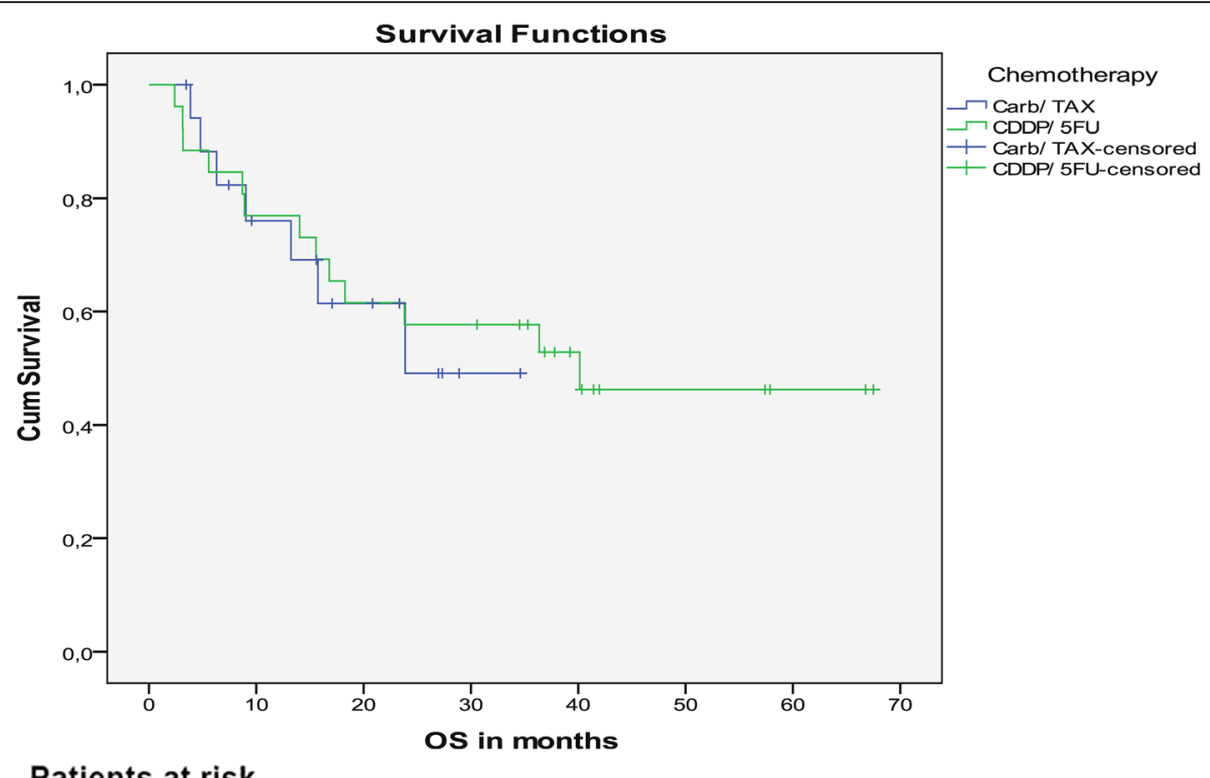

Patients at risk

Carboplatin/ Paclitaxel

$\begin{array}{llll}16.5 & 10 & 4.5 & 0.5\end{array}$

Cisplatin/ 5-fluoruracil

$\begin{array}{lllllll}26 & 20 & 16 & 12 & 6.5 & 3 & 1\end{array}$

Fig. 1 Overall survival. Patients at risk: Carboplatin/ Paclitaxel - 16.5, 10, 4.5, 0.5. Cisplatin/ 5-fluoruracil - 26, 20, 16, 12, 6.5, 3, 1.

toxicity in general as well as the rate of anemia, leukopenia and thrombocytopenia in particular was much higher than in other studies $[3,4,9]$. Nonetheless, toxicity results for patients treated with $\mathrm{CDDP} / 5 \mathrm{FU}$ match with the results by Tepper et al. [2] (57\% vs. 58\% of patients with hematologic $\geq \mathrm{III}^{\circ}$ toxicity) while two other studies report significant less hematologic toxicity in patients treated with cisplatin and 5-fluoruracil (15$17 \%)[4,8]$. However, because the difference in radiation dose between treatment groups was just 3.6 Gy our results underlines that the difference in hematologic toxicity is probably caused by the different chemotherapy regime. As in other studies leukopenia was the most common hematologic toxicity and our analysis showed a strong trend for higher grades of leukopenia in patients treated with $\mathrm{CDDP} / 5 \mathrm{FU}$ in the ITT-analysis and significantly higher grades of leukopenia in patients treated with $\mathrm{CDDP} / 5 \mathrm{FU}$ in the PP population.

Regarding non-hematologic side effects no significant difference was seen for the rate of insufficiencies of the anastomosis and the rate of in-hospitalmortality between both treatment regimes. However, the absolute difference in the rate of insufficiencies of the anastomosis is remarkable. While it was seen in $15 \%$ of patients who received $\mathrm{CDDP} / 5 \mathrm{FU}$, which is comparable to the results by Blom et al. [4] (13\% vs. $15 \%), 33 \%$ of patients treated with Carb/TAX were diagnosed with postsurgical insufficiency of the anastomosis. These results should be kept in mind and reviewed in further trials, especially because we don't have a convincing explanation.

In accordance with other studies no significant difference was seen for OS and FFR between both treatment groups [4, 9]. However, for patients treated with Carb/TAX median OS was significant shorter than in the CROSS-trial $[3,10]$. In the CROSS-trial median OS of patients with SCC was 81.6 months. In a retrospective trial the median OS for EC patients treated with Carb/TAX was not reached after 3 years [4]. Because OS after trimodal therapy is better for patients with SCC than for patients with AC, we have to point out that in this trial only $24 \%$ of patients had SCC. For patients treated with $\mathrm{CDDP} / 5 \mathrm{FU}$ median overall survival is comparable to other published results (17.7-53 months) [2, 4, 8, 11]. The short median OS of 17.7 months within the FFCD 9102 trial [11] (89\% SCC) is probably associated with the high rate of local treatment failure (34\%). Radiotherapy in this trial was done with either a conventional regime (46 Gy in 23 fractions) or as a split-course regime 


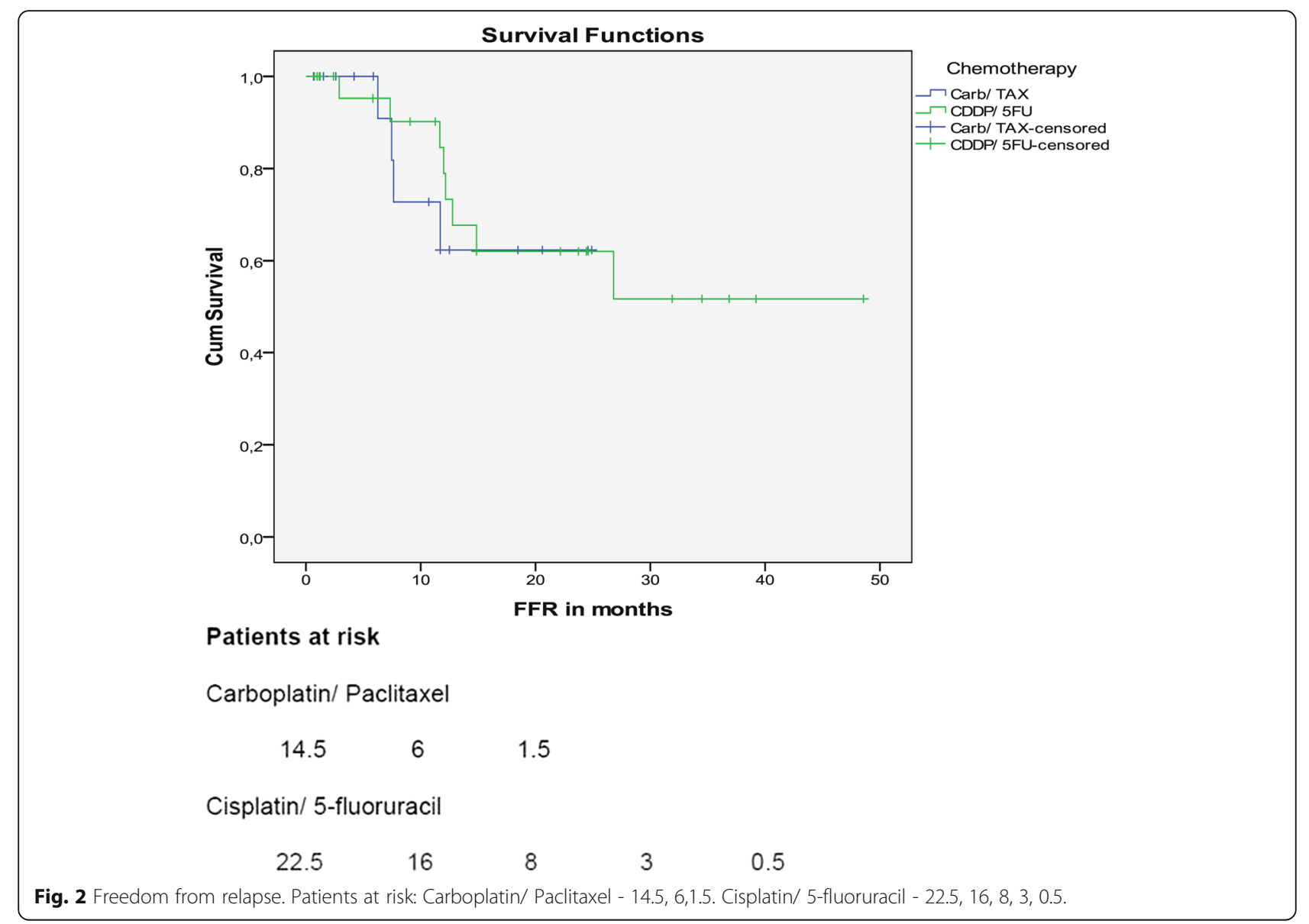

with 5 fractions and a daily dose of $3 \mathrm{~Gy}$ in the first and fourth week of treatment. In summary, the reason for the reduced median OS in patients treated according to the CROSS protocol remains unclear. However, when looking to the rate of local or distal disease progression our results show no increased incidence of treatment failure compared to the longterm data of the CROSS Trial [10]. Results for alternative chemotherapy regimens were reported in two further trials for patients undergoing definite chemoradiation for EC $[12,13]$. In a phase II trial by Wolf et al. [12] 135 patients (85\% SCC) received dCRT with mitomycin $\mathrm{C}$ and 5-fluoruracil. Radiation dose was more than $54 \mathrm{~Gy}$ in $85 \%$ of patients. Xia et al. [13] evaluated dCRT with weekly paclitaxel and 5fluoruracil and simultaneous radiotherapy (50.4 Gy or 61.2 Gy) for 53 patients (94\% SCC). Median OS was 15.6 months and 17.9 months, respectively. However, comparability is restricted, because patients were treated with dCRT instead of nCRT with subsequent surgery. While no further information about toxicity were given by Wolf et al. $\geq \mathrm{III}^{\circ}$ neutropenia was seen in $7 \%$ of patients treated with paclitaxel and 5fluoruracil [13].
In our study complete tumor regression (pCR, Becker Ia) was seen in 28\% (Carb/TAX) and 38\% (CDDP/5FU) of patients, respectively. These results match with the results of the retrospective trial published by Blom et al. and van Meerten et al. [4, 14]. While these studies included patients with SCC and AC (22-24\% SCC) studies focusing on only SCC patients report higher rates of pCR after nCRT with CDDP/5FU (51\%) or Carb/TAX (49\%) [3, 8]. This might be partially explained by the diagnostic tissue workup, because in our center we pursue a very rigorous course and pCR can only be stated when the whole tumor area has been subjected to histological workup. In accordance to the results of Blom et al., no statistically significant difference regarding the proportion of tumor regression grades was seen between both nCRT regimes [4]. An overview of different studies evaluating pCR, overall survival and hematologic toxicity of different nCRT regimes for SCC is given in Table 4.

In our study, all patients (100\%) undergoing nCRT with Carb/TAX and $71 \%$ of patients treated with CDDP/5FU received radiotherapy using VMAT. This is important, because different trials have already demonstrated the superiority of VMAT over 3D-CRT 


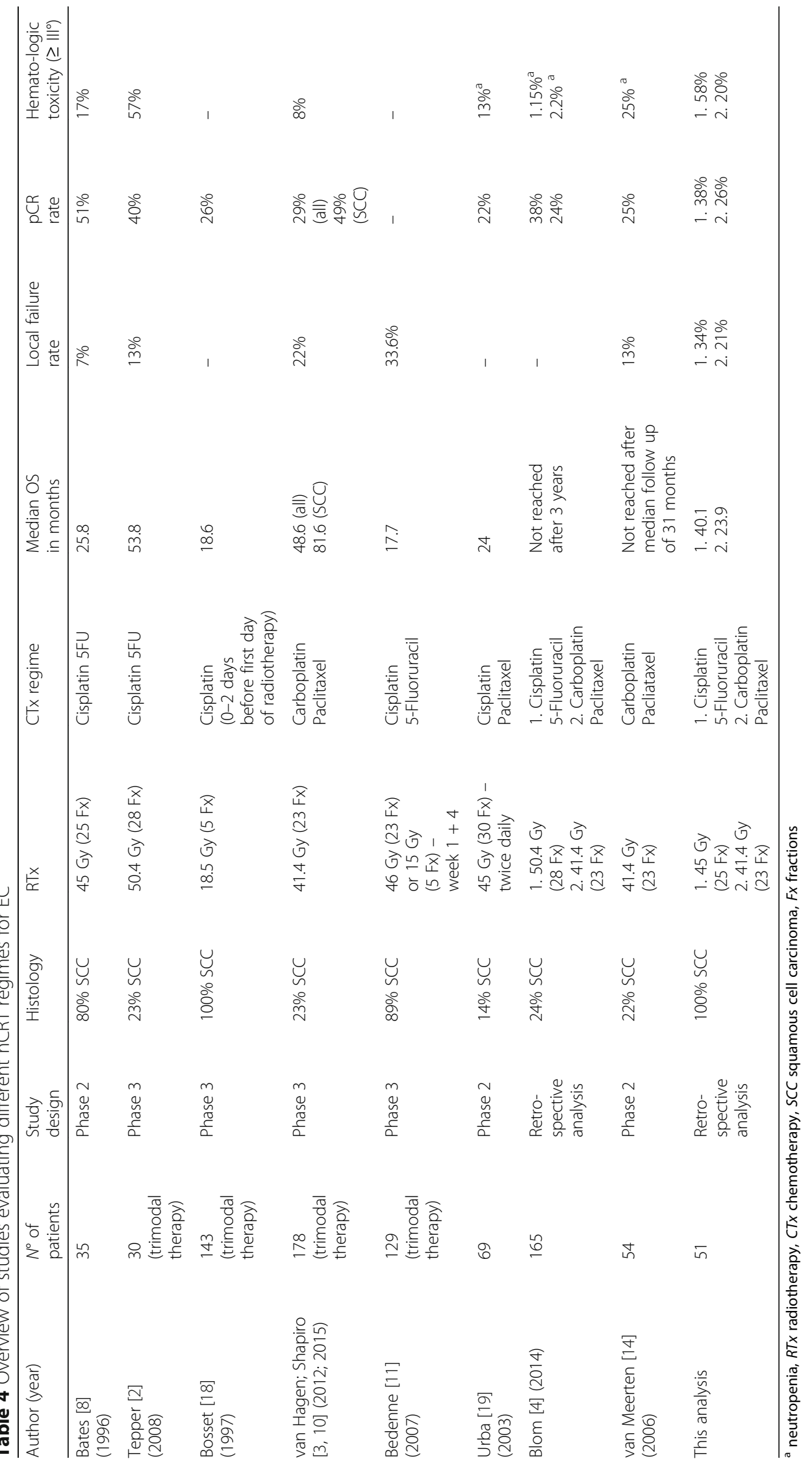


for radiotherapy of esophago-gastric cancer regarding dose reduction to the organs at risk when compared to 3D-CRT [15-17]. However, it remains questionable if these dosimetric differences alone can lead to higher rates of hematological side effects. In an analysis comparing VMAT and 3D-CRT for nCRT of esophageal cancer the use of VMAT was associated with higher rates of leukopenia when compared to 3D-CRT [16], but we have to point out that chemotherapy regime was not standardized. Therefore, the used chemotherapy regime seems to be more important for acute hematologic side effects.

Due to its retrospective nature this study has some limitations. The first problem is the relatively small number of patients in each treatment group. However, to the best of our knowledge this study includes the first dataset comparing nCRT with $\mathrm{CDDP} / 5 \mathrm{FU}$ or Carb/TAX for patients with only SCC. Another problem is the imbalance concerning follow-up time. Because nCRT with $\mathrm{CDDP} / 5 \mathrm{FU}$ was only used until 2014 and nCRT with Carb/TAX was only used since then, follow-up for patients treated with CDDP/5FU is obviously longer than for patient treated with Carb/PTAX, which can affect OS and FFR. Due to these limitations, the significance of the results is clearly impaired. So when looking to the fact that we could find no significant differences in terms of survival and treatment response between both groups, we have to keep in mind that the relatively small number of patients limits the power of the study.

\section{Conclusion}

In this retrospective analysis, no significant difference was seen for OS and FFR between nCRT with CDDP/ 5FU and nCRT with Carb/TAX. However, the application of $\mathrm{CDDP} / 5 \mathrm{FU}$ was associated with significantly more hematologic III $^{\circ}$ - toxicities compared to Carb/ TAX. Future prospective trials should investigate if these results are reproducible in randomized patient cohorts.

\section{Abbreviations}

3D-CRT: 3-dimensional conformal radiotherapy; AC: Adenocarcinoma; Carb/ TAX: Carboplatin and Paclitaxel; CDDP/5FU: Cisplatin and 5-fluoruracil; CTCAE: Common terminology criteria for adverse events; EC: Esophageal cancer; FFR: Freedom from relapse; Gy: Gray; ITT: Intention-to-treat; nCRT: neoadjuvant chemoradiation; OS: Overall survival; pCR: Pathologic complete response; PP: Per-protocol; SCC: Squamous cell carcinoma; VMAT: Volumetric modulated radiotherapy

\section{Acknowledgements}

Not applicable.

\section{Funding}

This research did not receive any specific grant from funding agencies in 448 the public, commercial, or not-for-profit sectors.

\section{Availability of data and materials}

The present data is summarized in this paper. The complete dataset can be retrieved from the authors upon formal request from interested readers.

\section{Authors' contributions}

SM developed the study design, collected, and interpreted data, performed statistical analysis and drafted the manuscript. SP made substantial contributions to conception and design of the study, interpreted data and revised the manuscript. MF, JSH, WW, HF and SC contributed significantly to the discussion and interpretation of the results $\mathrm{DH}$ made substantial contributions to conception and design of the study, analyzed and interpreted data and revised the manuscript. All authors read and approved the final manuscript.

Ethics approval and consent to participate

The study was performed in accordance with the ethics standards at the Technical University of Munich (TUM) (ethical vote $N^{\circ} 85 / 175$ ).

\section{Consent for publication}

Not applicable.

\section{Competing interests}

The authors declare that they have no competing interests.

\section{Publisher's Note}

Springer Nature remains neutral with regard to jurisdictional claims in published maps and institutional affiliations.

\section{Author details}

${ }^{1}$ Department of Radiation Oncology, Klinikum rechts der Isar, Technical University Munich, Ismaninger Str. 22, D-81675 Munich, Germany. ${ }^{2}$ German Cancer Consortium (DKTK), Partner Site Munich, Munich, Germany. ${ }^{3}$ Department of Surgery, Klinikum rechts der Isar, Technical University Munich, Ismaninger Str. 22, D-81675 Munich, Germany. ${ }^{4}$ Institute of Pathology, Klinikum rechts der Isar, Technical University Munich, Ismaninger Str. 22, D-81675 Munich, Germany. ${ }^{5}$ Institute of Innovative Radiotherapy (iRT), Helmholtz Zentrum München, Ingolstädter Landstraße 1, D-85764

Oberschleißheim, Germany.

Received: 1 April 2017 Accepted: 26 October 2017

Published online: 21 November 2017

\section{References}

1. Ferlay J, et al. Cancer incidence and mortality worldwide: sources, methods and major patterns in GLOBOCAN 2012. Int J Cancer. 2015;136(5):E359-86.

2. Tepper J, et al. Phase III trial of trimodality therapy with cisplatin, fluorouracil, radiotherapy, and surgery compared with surgery alone for esophageal cancer: CALGB 9781. J Clin Oncol. 2008;26(7):1086-92.

3. van Hagen $\mathrm{P}$, et al. Preoperative chemoradiotherapy for esophageal or junctional cancer. N Engl J Med. 2012;366(22):2074-84.

4. Blom RL, et al. Comparison of two neoadjuvant chemoradiotherapy regimens in patients with potentially curable esophageal carcinoma. Dis Esophagus. 2014;27(4):380-7.

5. Becker $\mathrm{K}$, et al. Histomorphology and grading of regression in gastric carcinoma treated with neoadjuvant chemotherapy. Cancer. 2003;98(7): 1521-30.

6. Ajani JA, et al. Esophageal and esophagogastric junction cancers, version 1. 2015. J Natl Compr Cancer Netw. 2015;13(2):194-227.

7. Porschen R. et al. [Not Available]. Z Gastroenterol. 2015 53(11): 1288-1347.

8. Bates BA, et al. Concurrent radiation therapy and chemotherapy followed by esophagectomy for localized esophageal carcinoma. J Clin Oncol. 1996; 14(1):156-63.

9. Honing J, et al. A comparison of carboplatin and paclitaxel with cisplatinum and 5-fluorouracil in definitive chemoradiation in esophageal cancer patients. Ann Oncol. 2014;25(3):638-43.

10. Shapiro J, et al. Neoadjuvant chemoradiotherapy plus surgery versus surgery alone for oesophageal or junctional cancer (CROSS): long-term results of a randomised controlled trial. Lancet Oncol. 2015;16(9):1090-8.

11. Bedenne $L$, et al. Chemoradiation followed by surgery compared with chemoradiation alone in squamous cancer of the esophagus: FFCD 9102. J Clin Oncol. 2007:25(10):1160-8.

12. Wolf $\mathrm{M}$, et al. Long-term outcome of mitomycin C- and 5-FU-based primary radiochemotherapy for esophageal cancer. Strahlenther Onkol. 2010;186(7):374-81. 
13. Xia Y, et al. A phase II study of concurrent chemoradiotherapy combined with a weekly paclitaxel and 5 -fluorouracil regimen to treat patients with advanced oesophageal carcinoma. Radiat Oncol. 2017;12(1):47.

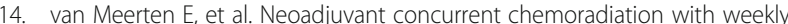
paclitaxel and carboplatin for patients with oesophageal cancer: a phase II study. Br J Cancer. 2006:94(10):1389-94.

15. Zhang $\mathrm{T}$, et al. Double-arc volumetric modulated therapy improves dose distribution compared to static gantry IMRT and 3D conformal radiotherapy for adjuvant therapy of gastric cancer. Radiat Oncol. 2015;10:114.

16. Munch $\mathrm{S}$, et al. Comparison of dosimetric parameters and toxicity in esophageal cancer patients undergoing 3D conformal radiotherapy or VMAT. Strahlenther Onkol. 2016;192(10):722-9.

17. Lin CY, et al. Dosimetric and efficiency comparison of high-dose radiotherapy for esophageal cancer: volumetric modulated arc therapy versus fixed-field intensity-modulated radiotherapy. Dis Esophagus. 2014; 27(6):585-90.

18. Bosset JF, et al. Chemoradiotherapy followed by surgery compared with surgery alone in squamous-cell cancer of the esophagus. N Engl J Med. 1997;337(3):161-7.

19. Urba SG, et al. Concurrent cisplatin, paclitaxel, and radiotherapy as preoperative treatment for patients with locoregional esophageal carcinoma. Cancer. 2003:98(10):2177-83.

\section{Submit your next manuscript to BioMed Central} and we will help you at every step:

- We accept pre-submission inquiries

- Our selector tool helps you to find the most relevant journal

- We provide round the clock customer support

- Convenient online submission

- Thorough peer review

- Inclusion in PubMed and all major indexing services

- Maximum visibility for your research

Submit your manuscript at www.biomedcentral.com/submit 